Relations industrielles

Industrial Relations

\title{
L'approche systémique en relations industrielles
}

\section{Viateur Larouche et Esther Déom}

Volume 39, numéro 1, 1984

URI : https://id.erudit.org/iderudit/050006ar

DOI : https://doi.org/10.7202/050006ar

Aller au sommaire du numéro

Éditeur(s)

Département des relations industrielles de l'Université Laval

ISSN

0034-379X (imprimé)

1703-8138 (numérique)

Découvrir la revue

Citer cet article

Larouche, V. \& Déom, E. (1984). L'approche systémique en relations industrielles. Relations industrielles / Industrial Relations, 39(1), 114-145. https://doi.org/10.7202/050006ar
Résumé de l'article

Les auteurs retiennent certains éléments pertinents à l'analyse systémique en relations industrielles et tentent de dégager une conception plus claire de ce champ d'étude.
Tous droits réservés (C) Département des relations industrielles de l'Université Laval, 1984
Ce document est protégé par la loi sur le droit d'auteur. L’utilisation des services d'Érudit (y compris la reproduction) est assujettie à sa politique d'utilisation que vous pouvez consulter en ligne.

https://apropos.erudit.org/fr/usagers/politique-dutilisation/ 


\title{
L'approche systémique en relations industrielles
}

\author{
Viateur Larouche \\ et
}

Esther Déom

Les auteurs retiennent certains éléments pertinents à l'analyse systémique en relations industrielles et tentent de dégager une conception plus claire de ce champ d'étude.

Les auteurs discutent la notion de relations industrielles par le truchement de l'approche systémique que l'on accole souvent à ce concept. Dans la première partie de cette étude, les différents éléments propres à la notion de système sont présentés et discutés. Les auteurs tiennent à souligner qu'il n'entre pas dans leur objectif de présenter une analyse exhaustive de la notion de système ou de la théorie générale des systèmes ${ }^{1}$. Leur but est plus modeste: il s'agit de retenir certains éléments jugés pertinents pour l'utilisation de l'approche systémique en relations industrielles. Dans la seconde partie, les auteurs appliquent ces caractéristiques à la notion de relations industrielles et essaient de dégager une conception plus claire de ce champ d'étude.

Le concept de système, développé à l'origine par les pères des sciences «exactes» est rapidement devenu un concept-clé dans toute discipline qui aspire au titre de science. Une brève revue de la littérature sur ce sujet suffit à nous en convaincre ${ }^{2}$. Les relations industrielles n'ont pas échappé à ce

* LAROUCHE, Viateur, professeur, École de relations industrielles, Université de Montréal.

DÉOM, Esther, étudiante au Ph.D., École de relations industrielles, Université de Montréal.

1 Pour une analyse de la pensée systémique, voir Pierre MONGEAU, «La pensée systémique. Historique et concepts», L'orientation professionnelle, vol. 16, no 6, avril 1981, pp. 33-50 et «La pensée systémique. Les concepts. Conclusion et perspectives», L'orientation professionnelle, vol. 17, no 1, juin 1981, pp. 9-52.

2 Les sciences ou disciplines récentes (informatique, communication, service social, robotique...) de même que les plus anciennes (chimie, biologie, mathématiques, physique...) se sont «mises» à l'approche systémique en vue d'obtenir une certaine intégration des connaissances propres à leur objet d'étude et développer une certaine capacité de prédire les variations de l'objet qui leur est propre. Par voie de conséquence, on voit ainsi apparaître dans presque tous les domaines le terme de «système». On y réfère autant pour désigner un système social, un système de transport en commun, un système d'information ou de mise en marché qu'un système comptable. Le concept est malheureusement utilisé trop souvent sans que l'on prenne vraiment le temps d'en préciser son contenu.

Relat. ind., vol. 39, no 1, 1984 (C) PUL ISSN 0034-379 X 
courant. Depuis que Dunlop ${ }^{3}$ a présenté son modèle alors que le concept venait tout juste d'être appliqué aux sciences naturelles, beaucoup d'autres théoriciens nous ont présenté leur vision d'un système de relations industrielles. Ces auteurs ont suivi la logique propre à l'approche systémique dont la première étape consiste à définir l'objet propre de chaque système. Ils s'y sont tellement concentrés qu'ils ont négligé de considérer d'autres caractéristiques qui façonnent cette notion, causant aussi beaucoup de confusion et une perception limitée des champs d'étude et disciplines auxquels ce concept a été appliqué.

Dans le présent article nous tenterons de comprendre d'abord ce qu'il faut entendre par "approche systémique». Pour ce faire, nous considérerons les principaux éléments qui caractérisent la notion de système. Les éléments qui sont généralement retenus pour préciser ce concept sont: 1- définition de système; 2 - objet d'un système (variable dépendante et indépendantes ou parties); 3- relations d'un système; 4- dimension opérationnelle; 5- dimension statique-dynamique; 6- dimension ouverte-fermée; 7-validité.

\section{La définition de système}

Avant de préciser ce qu'il faut entendre par système, il apparaît important de se demander pourquoi cette notion est si largement utilisée. Quels sont donc les avantages recherchés en utilisant l'approche systémique? On doit reconnaître que l'utilisation du mot «système» confère aux éléments ou aux idées auxquels on l'associe une certaine logique, un ordre, etc... C'est précisément cette qualité qui est recherchée par l'utilisation de l'approche dite «systémique». La plupart des théoriciens qui l'utilisent lui reconnaissent cette capacité d'intégrer et d'organiser des connaissances en un tout cohérent et ordonné. En effet, que ce soit au niveau des systèmes abstraits ou réels, des systèmes partiels ou généraux, en psychologie autant qu'en relations industrielles, l'approche systémique est génératrice d'un potentiel d'intégration des éléments ou des connaissances propres aux objets de ces disciplines. Heneman fait clairement ressortir cette qualité de l'approche lorsqu'il affirme que les relations industrielles ont un urgent besoin d'un «common conceptual model or paradigm of our industrial relations systems. Our new discipline in short is un-disciplined, disorganized and disordely» ${ }^{4}$.

3 John T. DUNLOP, Industrial Relations Systems, New York, Holt \& Co., 1958.

4 Herbert G. HENEMAN, «Conceptual System of Industrial Relations», Manpower and Applied Psychology, vol. 1 et 2, 1967, page 97. 
Ce potentiel intégrateur de l'approche systémique est également reconnu par Bertalanffy ${ }^{5}$ qui a cherché à intégrer l'ensemble des systèmes et à dégager les lois générales de ces systèmes. À un niveau moins global, on reconnaît également à l'approche systémique son utilité du fait qu'elle favorise la synthèse et l'intégration d'ensembles de connaissances. La notion «d'isomorphisme» ${ }^{6}$ représente en fait ce potentiel d'intégration, donc de généralisation, de la théorie générale des systèmes. Que ce soit Buckley ${ }^{7}$ dont l'intérêt se situe au niveau des systèmes socio-culturels, Heneman ${ }^{8}$ qui se préoccupe de donner aux relations industrielles un cadre d'analyse, Berrien $^{9}$ dont la préoccupation première est de fournir une base systémique à la psychologie sociale, Parsons ${ }^{10}$ qui insiste sur la nécessité de l'ordre ou même Forrester ${ }^{11}$ dont l'intérêt se situe plus spécifiquement au niveau de l'entreprise et de la prise de décision par le manager, on se rend bien compte que les notions d'ordre et d'organisation sont sous-jacentes à la définition même d'un système et, par le fait même, constituent des caractéristiques importantes de l'approche systémique.

D'une façon générale, bon nombre d'auteurs s'entendent pour définir un système comme un ensemble de variables reliées les unes aux autres et représentant les facettes propres d'un objet donné. Cette définition générale d'un «système», met l'accent sur l'interdépendance des parties d'un système. Heneman abonde dans ce sens lorsqu'il définit ce concept comme «a comprehensive set of variables in which all are united through some forms of regular interaction or interdependance» ${ }^{12}$. Buckley, dans la même perspective, considère un système comme «a complex of elements or components directly or indirectly related in a causal network» ${ }^{13}$.

5 Von L. BERTALANFFY, Théorie générale des systèmes, Paris, Dunod, 1973.

6 Selon MONGEAU, la définition de l'isomorphie peut être la suivante: deux systèmes sont isomorphes lorsqu'ils ont la même structure, c'est-à-dire lorsque toutes relations significatives existant entre deux éléments de l'un des systèmes se retrouvent entre les éléments correspondants de l'autre système», p. 16.

7 Walter BUCKLEY, Sociology and Modern Systems Theory, New-York, PrenticeHall, 1967.

8 HENEMAN, loc. cit.

9 Kenneth BERRIEN, General and Social Systems, Boston, Rutgers University Press, 1968.

10 Talcott PARSONS, The Social System, New-York, The Yale Press, 1951.

11 Jay W. FORRESTER, «Industrial Dynamics - After the First Decade», Management Science, vol. 14, no. 7, March 1968, pp. 398-415.

12 H.G. HENEMAN, D. YODER, Labor Economics, South Western Publishing Company, 1965 , p. 788 .

13 W. BUCKLEY, op. cit., p. 41. 


\section{Objet d'un système (variable dépendante et indépendantes ou parties)}

Un système étant défini comme un ensemble d'éléments en interaction, il importe maintenant de se demander selon quels critères un élément plutôt qu'un autre, peut être considéré comme faisant partie d'un système.

De façon générale, les éléments d'un système sont définis en termes de variables. La plupart des auteurs retiennent, à ce propos, deux (2) catégories (de base) de variables soient les variables dépendantes et indépendantes. Heneman, Buckley et Bertalanffy retiennent cependant une autre catégorie de variables qu'ils nomment "variable modératrice».

Dans ce contexte, la variable dépendante constitue le coeur d'un système, ce que l'on essaie d'expliquer ou encore ce que l'on essaie de comprendre..., bref l'objet même d'un système. Les variables indépendantes sont celles qui sont susceptibles d'expliquer la variable dépendante d'un système. Quant aux variables modératrices, elles affectent les relations qui existent entre les variables dépendantes et indépendantes. Leur effet doit cependant être assez important pour modifier les résultats de ces relations. Ainsi, tout élément, partie d'un système, doit être associé à l'objet même de ce système (variable dépendante - «out put») et permettre une meilleure compréhension de cet objet.

Certains auteurs n'insistent pas sur cette dimension importante (partie) d'un système. Berrien ne retient comme caractéristique essentielle d'une composante d'un système, que le fait qu'elle interagisse avec une autre partie du système pour produire un résultat qui se distingue des interactions elles-mêmes et des entrées (inputs). Il ne retient que l'aspect fonctionnel d'une composante.

\section{Les relations d'un système}

Les relations entre les parties (variables) d'un système constituent une des principales caractéristiques de cette entité. En effet, on ne peut considérer un système comme étant composé que d'une simple liste d'éléments (ou de parties) sans aucune relation entre eux (elles). Il faut que ces éléments puissent constituer un tout cohérent, bref que des relations existent entre les variables. On peut cependant discuter longuement le genre de relations qui devraient exister entre les variables que l'on retrouve à l'intérieur d'un système. Heneman est d'avis qu'un système devrait comprendre trois (3) types de relations:

- les relations causales qui fournissent des explications sur la raison d'être d'une situation observée; 
- les relations fonctionnelles qui suggèrent comment les variables sont associées entre elles, et

- les relations séquentielles qui font surface lorsque des variables ont un effet l'une après l'autre de façon ordonnée.

Les relations causales, comme leur nom le laisse suggérer, indiquent une relation de cause à effet, d'une variable indépendante avec la variable dépendante. C'est ce type de relation que l'on cherche le plus souvent à établir et c'est celui qu'Heneman retient comme le plus important. Cependant, cette relation est également la plus difficile à démontrer.

La relation causale a cependant été beaucoup critiquée notamment par Buckley, qui souligne l'incapacité des modèles simples de causalité ou de corrélation à expliquer des systèmes sociaux: «a simple model of causation and correlation and its methodology is woefully inadequate in the face of complex adaptive systems $)^{14}$. La complexité des systèmes sociaux ne peut donc être rendue qu'à travers un autre type de relations que Buckley nomme les chaînes causales circulaires. Ce nouveau type constitue en fait un raffinement des relations réciproques ou mutuelles, approche qui reconnaît l'interdépendance mutuelle de certaines parties d'un système.

La causalité retenue par Buckley diffère totalement par l'introduction du concept de rétro-action emprunté à la cybernétique, de la relation causale traditionnelle. Cette causalité circulaire rejoint celle retenue par Forrester qui reconnaît également l'essentialité de la rétro-action et insiste sur l'interaction continue entre le niveau et le degré des variables.

Il semble ainsi y avoir un certain consensus sur l'utilité des relations non-linéaires dans les systèmes sociaux complexes plutôt que de relations causales à sens unique. Heneman est le seul à affirmer l'importance des relations de ce dernier type. D'autres auteurs, tels Berrien et Parsons, ne traitent simplement pas de ce point. Le premier retient bien sûr le concept de rétro-action mais en l'appliquant aux extrants et intrants du système. Quant à Parsons, même si ce point ne semble pas retenir son attention, on peut déduire, à l'analyse de son modèle, qu'il privilégie les relations de type fonctionnel.

Il nous semble cependant, en dépit des critiques formulées à l'encontre de la relation de causalité simple, à sens unique, que c'est encore ce type de relation qui fournit le plus d'indication sur les liens entre variables dépendantes et variables indépendantes. Les relations causales circulaires sont également utiles, car il va de soi qu'une variable dépendante dans un système peut se retrouver comme variable indépendante dans un autre système et les relations peuvent au total, prendre l'aspect de chaînes causales circulaires.

$14 \quad$ Ibid., p. 67. 
Le graphique I, nous permet de mieux saisir cette différence.

\section{GRAPHIQUE I}

Relations de type causale et causale circulaire

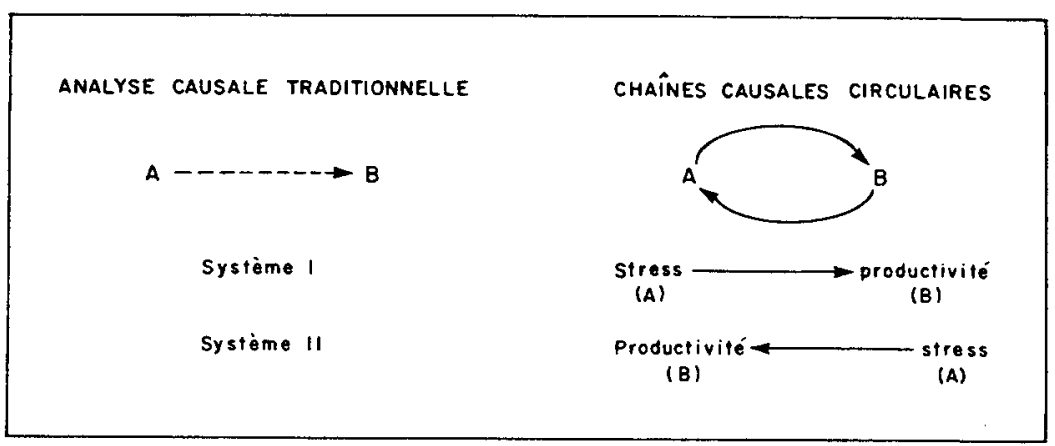

\section{Dimension opérationnelle}

Nous avons jusqu'à maintenant fait porter notre attention sur des systèmes conceptuels et abstraits. Pourtant, il existe, comme le souligne si justement Heneman, une autre dimension d'un système, soit son aspect opérationnel qui appartient au domaine du réel, des faits et qui complète celui des concepts. Heneman est le seul qui montre clairement les liens qui existent entre ces deux parties d'un système et par quel processus on passe de l'un à l'autre. À ce sujet, il propose une logique déductive allant du système au modèle, de la théorie aux hypothèses pour finalement atteindre la réalité ou encore les faits. Le diagramme qui suit nous permet de mieux visualiser ce cheminement.

Brièvement, voyons comment sont définis les éléments principaux de ce diagramme.

Système: Un système consiste en un ensemble de variables en interaction ou en interdépendance les unes avec les autres.

Modèle: D'un niveau aussi abstrait que le système, le modèle est plus spécifique et plus limité. Il est constitué également d'un ensemble de variables reliées entres elles d'une façon plus spécifique. Bref, il s'agit en quelque sorte d'un sous-système. 
GRAPHIQUE 2

Démarche associée à la logique scientifique approche inductive et déductive

\begin{tabular}{|c|c|c|c|}
\hline $\begin{array}{l}\text { NIVEAU } \\
\text { D'ABSTRACTION }\end{array}$ & $\begin{array}{c}\text { PORTÉE DES } \\
\text { RELATIONS ÉTABLIES }\end{array}$ & VARIABLES & TYPE DE SYSTĖME \\
\hline 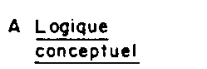 & & $\begin{array}{l}\text { entièrement } \\
\text { conceptuelles }\end{array}$ & \multirow{4}{*}{$\begin{array}{c}\text { Systeme } \\
\text { conceptuel }\end{array}$} \\
\hline \multirow{2}{*}{$\begin{array}{l}\text { a) definition } \\
\text { b) description } \\
\text { c) clossiticotion }\end{array}$} & Système & \multirow{2}{*}{$\begin{array}{l}\text { scientifiques } \\
2 \text { ou plus } \\
\text { conceptuelles }\end{array}$} & \\
\hline & I & & \\
\hline & Modèle & scientifiques & \\
\hline B $\frac{\text { Réolité }}{\text { operationnel }}$ & & opérotionnelles & \\
\hline $\begin{array}{l}\text { a) definition } \\
\text { b) description } \\
\text { c) clossificotion }\end{array}$ & Hypothèses & $\begin{array}{c}\text { i.e. } \\
\text { ciassifiées }\end{array}$ & $\begin{array}{c}\text { système } \\
\text { opérationnel }\end{array}$ \\
\hline & & 2 ou plus & \\
\hline & Faits & $\begin{array}{l}\text { catégorielles } \\
\text { ou ad-hoc }\end{array}$ & \\
\hline
\end{tabular}

Théorie: C'est un modèle qui possède une spécificité telle qu'il est possible de le tester empiriquement. Une théorie doit être définie opérationnellement ce qui lui permet de faire le lien entre le monde de l'abstrait et du concret. Bref, une théorie constitue une base duquelle découle une ou plusieurs hypothèses.

Hypothèse: C'est une tentative d'explication de la réalité. En d'autres mots, l'hypothèse est une affirmation qui se rattache à un phénomène réel et qui demande à être vérifiée ou encore testée.

On remarque que le système et le modèle appartiennent au domaine conceptuel et que l'hypothèse et les faits appartiennent au monde du réel. La théorie assure le lien entre ces deux niveaux d'abstraction. Le diagramme précédent nous montre également la démarche à suivre lorsque l'on adopte une approche logico-déductive pour passer de l'abstrait aux faits et l'inverse lorsque l'on opte pour une approche inductive c'est-à-dire une démarche qui va des faits vers l'abstrait. 
À part Heneman, Forrester est le seul à s'être vraiment préoccupé de l'aspect opérationnel d'un système. Selon lui les variables et leurs relations doivent pouvoir être quantifiables pour être utilisées. Il y arrive par l'utilisation de la simulation qui permet d'atteindre la réalité par le truchement de définitions et de boucles de rétroaction.

\section{Dimension statique-dynamique}

Un système est dynamique ${ }^{15}$ lorsqu'il tient compte du changement et de l'évolution dans le temps. Il est statique lorsqu'il ne fait référence qu'à une situation «arrêtée» dans le temps. Sur ce plan, un système doit tenir compte du changement. Parsons propose un système statique. Son approche affirme la primauté de l'équilibre, de l'ordre, de l'adaptation sur le changement, et ne retient que les structures dominantes et légalisées comme composantes du système. Il présente un modèle qui ne tient pas compte des structures déviantes qui, finalement intégrées, sont qualifiées de dysfonctionnelles.

À l'opposé de Parsons, Buckley affirme la primauté du changement sur la structure. Il se montre particulièrement critique à l'endroit de Parsons:

«In sum, we note that the model leads only to a consideration of such mechanisms as those of defense, adjustment and deviance. Control, all aimed at adaptation of the actor to a given dominant structure, with no consideration given to the historically obvious mechanisms that adapt or change the system structure to accomodate the actor and maintain the total system. ${ }^{16}$

Heneman, pour sa part, n'est pas très clair sur cet aspect d'un système bien que l'on puisse déduire de ses écrits qu'il considère un système comme devant être essentiellement dynamique. Forrester, par contre, se préoccupe uniquement des systèmes dynamiques puisque par définition la dynamique industrielle concerne l'étude des phénomènes dans le temps.

\section{Dimension ouverte-fermée}

Mongeau retient 3 types de systèmes: le système isolé qui n'a aucun échange avec l'environnement; le système fermé qui reçoit de l'énergie de l'extérieur mais qui ne possède pas de rétro-action permettant l'ajustement du système; le système ouvert enfin, est en relation constante avec l'environnement et s'ajuste. Mongeau retient également les caractéristiques de Katz et Kahn pour désigner un système ouvert.

15 Selon MONGEAU, le «statique» et le «dynamique» sont les deux aspects qui nous permettent d'analyser l'organisation d'un système, le statique correspond aux structures du système et le dynamique, aux fonctions du système.

16 BUCKLEY, op. cit., p. 30. 
Selon Katz et Kahn ${ }^{17}$, un système ouvert se caractérise par l'importation d'énergie venant de l'environnement, sa transformation en un produit propre au système et l'exportation de ce produit dans l'environnement. Les systèmes ouverts s'identifient également par les phénomènes d'entropie ${ }^{18}$ négative, de rétro-action, d'homéostasie, de différentiation et d'équifinalité.

Ces éléments constituent, de façon générale, les différents aspects retenus par les auteurs qui insistent sur l'aspect ouvert-fermé d'un système. Le modèle parsonien caractérisé par la recherche de l'équilibre, ne fait référence qu'à un système fermé. Par contre Buckley reconnaît qu'un système social doit essentiellement être un système ouvert, non pas uniquement à cause de l'échange continu avec l'environnement mais surtout parce que cet échange est un facteur essentiel à la viabilité d'un système. Berrien ajoute sur ce point que tous les systèmes sont ouverts mais qu'ils le sont en fonction d'une certaine gradation. En raisonnant de la sorte, il reconnaît l'existence de niveaux rejoignant ainsi Heneman et Buckley qui conçoivent également l'ouverture d'un système en terme de niveaux. Sur ce plan, Heneman avance qu'il peut exister plusieurs catégories de buts dans un système du fait que l'on tienne compte des différents niveaux: celui de l'individu, du groupe et de la société. Ainsi, plusieurs systèmes conceptuels sont possibles à chacun de ces niveaux.

L'ouverture du système peut également être comprise dans un autre sens. Forrester, s'intéressant à la dynamique industrielle, reconnaît l'existence de variables exogènes qui ne sont pas essentielles au système. Cependant, lorsqu'elles le deviennent, elles sont alors intégrées au système.

Bref, sauf Parsons, tous reconnaissent qu'un système ne peut qu'être ouvert et que cette caractéristique permet un échange continu avec l'environnement, essentiel à la viabilité du système.

\section{La validité}

La plupart des auteurs relient cet élément à l'aspect opérationnel du système. Pour bon nombre d'auteurs, la validité d'un système réside dans sa capacité à rendre compte adéquatement de la réalité. Ainsi chez Forrester, le test d'un système réside dans la possibilité d'en quantifier les éléments et dans sa capacité à représenter une situation réelle.

17 D. KATZ and R.L. KAHN, The Social Psychology of Organisations, New York, Wiley \& Sons, 1966.

18 L'entropie fait référence au processus de détérioration d'un objet (ici d'un système) lorsqu'il n'est pas revivifié. 
«The degree to which the model behaves like the actual system that is being modelled is one measure of model validity. ${ }^{19}$

Heneman croit cependant qu'un système peut être valide en soi et non par rapport à sa capacité d'expliquer la réalité. Un système conceptuel se définissant essentiellement par les variables dépendantes et indépendantes et les relations qui existent entre elles, on peut en déduire que plus ces relations sont définies et mieux elles expliquent une variation de la variable dépendante, plus le système est valide. Buckley abonde également dans ce sens.

Nous croyons ainsi qu'un système conceptuel doit d'abord se définir par sa logique interne. Il ne peut bien sûr échapper au test de la confrontation avec la réalité mais ce n'est pas la première étape qui doit être franchie. À cet égard, le schéma d'Heneman (Graphique 2) illustre bien les différentes étapes à franchir pour passer du niveau conceptuel au niveau opérationnel.

Le Tableau 1 résume la position des auteurs étudiés relativement aux différentes caractéristiques propres à l'approche systémique.

On remarque d'abord qu'aucun auteur ne traite simultanément de tous les aspects identifiés. Il ne s'agit pas ici d'accorder une palme d'or à l'auteur le plus méritant mais tout simplement de constater qu'il existe encore à l'heure actuelle et en dépit de l'utilisation de la notion de système depuis déjà quelques décennies, un manque certain de connaissances de ces caractéristiques particulières. On peut néanmoins dégager certaines tendances. Par le truchement des éléments que les auteurs retiennent pour caractériser un système, on note qu'un système est considéré comme un ensemble d'éléments en interaction. Ces éléments ou variables sont généralement classés en deux catégories soient les variables dépendantes et indépendantes. Les conditions d'admission des éléments d'un système sont très variées, quand elles sont précisées.

Quant aux relations entre les parties d'un système, on peut retenir un certain consensus sur l'utilité d'utiliser des relations non linéaires.

Laurent Bélanger ${ }^{20}$, utilisant une nomenclature quelque peu différente, identifie les principales composantes d'un système comme suit:

1- les résultats recherchés - «out put»

2- les activités

3- les ressources (inputs ou entrées)

4- la rétroaction ou le retour d'information

5- la distribution

6- l'environnement d'un système

19 FORRESTER, op. cit., p. 414.

20 Laurent BÉLANGER, Gestion des ressources humaines, Chicoutimi, Gaétan Morin, 1978, p. 14. 
TABLEAU 1

Caractéristiques de l'approche systémique selon certains auteurs

\begin{tabular}{|c|c|c|c|c|c|c|}
\hline Auteurs & $\begin{array}{c}\text { Objet } \\
\text { (variable) } \\
\text { (dependante) } \\
\text { («out putm) }\end{array}$ & Relations & $\begin{array}{c}\text { Aspect } \\
\text { opérationnel }\end{array}$ & $\begin{array}{l}\text { Dynamique } \\
\text { ou statique }\end{array}$ & $\begin{array}{l}\text { Ouvert } \\
\text { fermé }\end{array}$ & Validité \\
\hline $\begin{array}{l}\text { Heneman } \\
\text { (système de } \\
\text { relations ind.) }\end{array}$ & $\begin{array}{l}\text { Variables: } \\
\text { - dépendantes } \\
\text { - indépendantes } \\
\text { - modératrices. }\end{array}$ & Causales & $\begin{array}{l}\text { Distingue } \\
\text { entre syst. } \\
\text { conceptuel et } \\
\text { opérationnel et } \\
\text { montre la dyna- } \\
\text { mique entre eux. }\end{array}$ & ${ }^{*} \mathrm{~N} / \mathrm{P}$ & $\begin{array}{l}\text { Ouverture } \\
\text { exprimée par le } \\
\text { concept de } \\
\text { niveaux du } \\
\text { système }\end{array}$ & $\begin{array}{l}\text { Pour un système } \\
\text { conceptuel: } \\
\text { logique et } \\
\text { cohérence. }\end{array}$ \\
\hline $\begin{array}{l}\text { Buckley } \\
\text { (système socio- } \\
\text { culturel) }\end{array}$ & Idem & $\begin{array}{l}\text { Chaînes } \\
\text { causales et } \\
\text { circulaires. }\end{array}$ & $N / P$ & $\begin{array}{l}\text { Dynamique } \\
\text { mais n'en pré- } \\
\text { cise pas le sens. }\end{array}$ & $\begin{array}{l}\text { Ouvert: } \\
\text { l'èchange avec } \\
\text { l'environnement } \\
\text { est essentiel } \\
\text { à la viabilité } \\
\text { du système. }\end{array}$ & $\begin{array}{l}\text { Plus les rela- } \\
\text { tions sont pré- } \\
\text { tcises, plus le sys- } \\
\text { tème est valide. }\end{array}$ \\
\hline $\begin{array}{l}\text { Bertalanffy } \\
\text { (les systèmes } \\
\text { en général) }\end{array}$ & Idem & $\begin{array}{l}\text { Interaction mu- } \\
\text { tuelle. N'en } \\
\text { précise pas le } \\
\text { sens. }\end{array}$ & $\mathbf{N} / \mathbf{P}$ & $N / P$ & $\begin{array}{l}\text { Reconnaît } \\
\text { l'existence d'une } \\
\text { hiérarchie de } \\
\text { systèmes. }\end{array}$ & $\mathrm{N} / \mathrm{P}$ \\
\hline $\begin{array}{l}\text { Parsons } \\
\text { (système social) }\end{array}$ & $\begin{array}{l}\text { Parties du sys- } \\
\text { tème confon- } \\
\text { dues avec la } \\
\text { structure domi- } \\
\text { nante. }\end{array}$ & $\mathbf{N} / \mathbf{P}$ & $\mathbf{N} / \mathbf{P}$ & $\mathrm{N} / \mathrm{P}$ & Fermé & $\begin{array}{l}\text { Confrontation } \\
\text { avec la réalité }\end{array}$ \\
\hline $\begin{array}{l}\text { Forrester } \\
\text { (dynamique } \\
\text { industrielle) }\end{array}$ & $\begin{array}{l}\text { Variables: } \\
\text { - dépendantes } \\
\text { - indépendantes }\end{array}$ & $\begin{array}{l}\text { Relations non- } \\
\text { linéaires } \\
\text { (interaction) } \\
\text { continue). }\end{array}$ & $\begin{array}{l}\text { Privilégie } \\
\text { l'aspect opéra- } \\
\text { tionnel. }\end{array}$ & $\begin{array}{l}\text { Dynamique } \\
\text { mais n'en pré- } \\
\text { cise pas le sens. }\end{array}$ & Fermé & $\begin{array}{l}\text { Confrontration } \\
\text { avec la réalité }\end{array}$ \\
\hline $\begin{array}{l}\text { Berrien } \\
\text { (psychologie } \\
\text { sociale) }\end{array}$ & $\mathrm{N} / \mathrm{P}$ & $\mathrm{N} / \mathrm{P}$ & $N / P$ & $\mathrm{~N} / \mathrm{P}$ & $\begin{array}{l}\text { Tous les sys- } \\
\text { tèmes sont } \\
\text { ouverts. Il existe } \\
\text { des degrès d'ou- } \\
\text { verture. }\end{array}$ & $\begin{array}{l}\text { Confrontation } \\
\text { avec la réalité } \\
\text { - }\end{array}$ \\
\hline
\end{tabular}

*N/P: non précisé 
$\mathrm{Si}$ on applique ces composantes aux caractéristiques de l'approche systémique retenues par les auteurs étudiés on note que la majorité d'entre eux tiennent compte de l'objet d'un système (les résultats recherchés - «out put») ainsi que des relations (les activités) qui doivent exister entre les parties d'un système.

L'aspect opérationnel (la rétroaction ou le retour d'information) renvoie à la distinction existant entre les systèmes conceptuels et opérationnels et touche aussi, dans l'esprit de plusieurs auteurs, à la validité d'un système, c'est-à-dire sa capacité à représenter adéquatement la réalité.

L'ouverture d'un système (l'environnement) peut être définie par l'échange continu avec l'environnement. Cet échange est essentiel à la viabilité du système (Buckley). Il peut être vu en terme de niveaux (Heneman et Buckley), ou en termes de degrés (Berrien).

Quant au dynamisme d'un système (les ressources - inputs ou entrées) on fait référence à sa capacité d'intégrer le changement.

Enfin, les auteurs retenus soulignent que la validité (distribution) d'un système s'appuie sur sa capacité à acheminer dans l'environnement son «produit».

\section{L'APPROCHE SYSTÉMIQUE EN RELATIONS INDUSTRIELLES}

La première partie de cet article a fait ressortir les principales caractéristiques associées à la notion de système. Il s'agit donc, dans cette seconde partie, de les appliquer aux relations industrielles afin de voir si cette «discipline» encore à l'état naissant, peut se voir appliquer cette notion. L'exercice est important car les balises qui sont associées à l'approche systémique permettront de préciser le concept de relations industrielles faisant ressortir la force ou la précarité des traits propres à cette expression. Le lecteur comprendra que les auteurs ne pourraient dans un article, présenter, comparer et critiquer la pensée de chaque auteur qui s'intéresse aux relations industrielles. Notre objectif est d'abord de livrer au lecteur les résultats de nos recherches et de nos réflexions, résultats qui s'articulent autour des caractéristiques de la notion de système retenus en première partie pour ensuite proposer une démarche qui permettrait de clarifier notre champ d'étude.

L'ambiguité qui règne autour de l'objet propre des relations industrielles, note Delorme, fait que l'on détecte une série d'opinions les plus diverses lorsqu'on questionne l'identité propre des relations industrielles. L'un des 
problèmes majeurs réside en ce qu'on n'a pas encore réalisé l'osmose des disciplines auxquelles l'on a recours pour circonscrire l'objet formel. ${ }^{21}$

Le temps semble maintenant venu pour un nouvel effort de clarification de ce champ d'étude. Plusieurs auteurs ont déjà lancé un cri de ralliement. Somers ${ }^{22}$, Heneman et Kochan ${ }^{23}$, pour ne mentionner que ceux-ci, soulignent qu'il est temps d'ordonner et de circonscrire ce nouveau champ d'étude. Plus précisément, ils n'hésitent pas à affirmer que les relations industrielles ont besoin d'un cadre conceptuel qui pourrait s'avérer utile pour coordonner les initiatives de recherche, ordonner la connaissance et offrir un terrain de rencontre pour les chercheurs de différentes disciplines qui s'intéressent de près ou de loin aux problèmes de relations industrielles. Delorme note avec beaucoup d'à propos, que «l'absence d'un cadre théorique unificateur en relations industrielles, le recours fréquent à des méthodologies issues d'autres sciences de l'homme donnent à penser que les relations industrielles constituent encore un champ d'étude dont les bornes restent à délimiter en fonction d'assises théoriques plus solides». ${ }^{24}$

\section{Les éléments d'un système de relations industrielles}

Malgré l'effort fourni depuis plusieurs décennies pour définir l'objet d'étude des relations industrielles, on se doit de constater qu'il n'existe pas encore de consensus à ce sujet. La définition d'un objet-clef (variable dépendante) constitue pourtant la première étape dans la construction d'un système. Tous les auteurs intéressés aux relations industrielles n'utilisent cependant pas l'approche systémique telle que nous l'avons déjà présentée. Beaucoup utilisent l'expression système de relations industrielles sans jamais préciser ce qu'ils entendent par cette expression. Aussi, nous ne présenterons dans cette partie que ceux qui précisent leur conception des relations industrielles en utilisant au moins deux éléments de l'approche systémique.

Deux courants d'idées regroupent la majorité des théoriciens en relations industrielles sur cette question. Un premier groupe définit le système de relations industrielles comme celui qui permet la création ou l'adminis-

21 F. DELORME, Élaboration d'un sous-système en relations industrielles, (texte miméographié), École de relations industrielles, Université de Montréal, décembre 1972, p. 3.

22 G.G. SOMERS, «Bargaining Power and Industrial Relations Theory», G.G. Somers (Ed.), Essays in Industrial Relations Theory, Iowa, State University Press, 1969, pp. 39-55.

23 H.G. HENEMAN, «Toward a General Conceptual System of Industrial Relations: How do we get there?», G.G. Somers (Ed.), Essays in Industrial Relations Theory, Iowa, Iowa State University Press, 1969, pp. 3-24. T.A. KOCHAN, Collective Bargaining and Industrial Relations, Homewood, Ill., Irwin, 1980, p. 18.

24 F. DELORME, Étude empirique de la satisfaction au travail chez les contremaîtres québécois, thèse de maîtrise en relations industrielles, Université de Montréal, 1974, p. 5. 
tration des règles régissant les relations du travail. Le second groupe insiste plutôt sur l'identification d'un objet d'étude qui graviterait autour des «ressources humaines» ou encore «la main-d'oeuvre».

Les tenants du premier courant mettant l'accent sur les règles ou si l'on préfère sur le «contenant» ou la «forme», tandis que ceux qui adhèrent à la seconde tendance insistent sur le fait que tout système doit d'abord être constitué autour d'un objet d'étude ou d'analyse et, à ce propos, ils proposent comme cible les ressources humaines ou encore la main-d'oeuvre.

L'initiateur du premier courant et celui qui a introduit la perspective des systèmes dans l'étude des relations industrielles est bien sûr Dunlop ${ }^{25}$. Bien que critiqué depuis presque sa parution, le modèle de Dunlop a néanmoins servi de point de départ à plusieurs études ultérieures et les auteurs qui s'en sont servi ont surtout tenté de l'améliorer sans le rejeter ${ }^{26}$.

Selon Dunlop, un système de relations industrielles «is to be viewed as an analytical subsystem of an industrial society on the same logical plane as an economic system, regarded as another analytical subsystem ${ }^{27}$. Ce système comprend des acteurs (les travailleurs et leurs organisations, les employeurs et leurs organisations, l'État et ses agences spécialisées), des contextes considérés comme des données (technologie, marché, pouvoir), une idéologie commune aux acteurs et qui contribue au maintien du système enfin, la création de règles qui gouvernent ces acteurs.

Le fait pour Dunlop d'insister à certains moments sur la création de règles ${ }^{28}$ et à certains autres sur leur administration a soulevé une certaine confusion sur ce qu'il considérait comme l'objet d'étude des relations industrielles $^{29}$.

25 DUNLOP, loc. cit.

26 Allan FLANDERS, Industrial Relations: What is Wrong with the System?, London, Faber, 1965. A.J.N. BLAIN, J. GENNARD, «Industrial Relations Theory: A Critical Review», British Journal of Industrial Relations, Vol. VIII, No. 3, novembre 1970, pp. 389-407. CRAIG, Alton, «A Model for the Analysis of Industrial Relations», H.C. Jain (ed.), Canadian Labour \& Industrial Relations, Public and Private Sectors, Toronto, McGraw-Hill, 1974, pp. 2-12. KERR, C., DUNLOP, J.T., HARBISON, F. and C.A. MYERS, Industrialism and Industrial man, Cambridge, Harvard University Press, 1960.

27 DUNLOP, op. cit., p. 5.

28 Voir à ce propos J.F.B. GOODMAN and als, «Rules in Industrial Relations Theory: A Discussion», Industrial Relations Journal, Vol. 6, no. 1, 1975, pp. 14-30.

29 Le terme relations industrielles a fait son apparition aux États-Unis en 1912, au Congrès. L'expression a été par la suite popularisée en Grande-Bretagne et fut utilisée pour la première fois de façon officielle dans le «Survey of Industrial Relations» publié par le ministère du Commerce et de l'Industrie en 1926. 
L'approche de Dunlop a été acceptée par plusieurs adeptes. Elle a également eu ses «critiques». Parmi les premiers, on retrouve Flanders ${ }^{30}$ qui a été en Angleterre le propagateur de cette conception des relations industrielles. Ce dernier retient essentiellement la même variable dépendante que Dunlop. Dans la même ligne d'idée, Bain et Clegg qui ne précisent pas le contenu du concept de système, retiennent comme objet d'étude des relations industrielles l'ensemble des règles, institutions et processus, formels et informels, structurés ou non structurés mis en place pour encadrer la relation d'emploi -job regulation ${ }^{31}$. Cette définition élargit cependant l'idée originale de Dunlop en incluant les processus et les structures non-dominantes comme variables. On doit également mentionner l'ouvrage de Kerr, Dunlop, Harbison et Myers ${ }^{32}$ portant sur l'étude de différents systèmes de relations industrielles, centré sur la fonction de structuration de la main-d'oeuvre en situation d'industrialisation, fait ressortir le déterminisme des forces économiques et technologiques par rapport à cette fonction.

Blain et Gennard ${ }^{33}$ ont également tenté de raffiner le modèle original de Dunlop en incluant les processus et les variables de la personnalité négligés par celui-ci. Ils avancent la formulation mathématique suivante: $r: f(a, t, e$, $\mathrm{s}$, i) où $\mathrm{r}$ : règles, a: acteurs, $\mathrm{t}$ : contexte technologique, e: contexte de marché ou contraintes budgétaires, $s$ : contexte de pouvoir et $i$ : idéologie du système. Les auteurs tiennent compte du processus de détermination des règles en comparant le système de relations industrielles à deux moments différents: $t_{1}$ (situation originale) et $t_{2}$ (situation modifiée). Ils concluent que cette façon de faire permet d'intégrer les processus de détermination des règles comme variable dépendante, ce qui rend le modèle dynamique. Ils ajoutent enfin une variable indépendante $x$, qui représente les facteurs de la personnalité. Leur équation finale se présente comme suit:

$$
p: f(r, a, t, e, s, i, x)
$$

Blain et Gennard identifient des variables importantes, et précisent que les relations entre ces variables doivent être de nature causale.

Craig $^{34}$ et Singh ${ }^{35}$ ont également introduit des améliorations importantes au modèle original de Dunlop. Craig a ainsi élaboré un modèle composé de quatre éléments:

30 FLANDERS, loc. cit.

31 BAIN, G.S. and H.A. CLEGG, "A Strategy for Industrial Relations Research in Great Britain", British Journal of Industrial Relations, Vol. XII, No. 1, 1974, p. 7.

32 KERR et als, loc. cit.

33 BLAIN and GENNARD, op. cit., pp. 401-407.

34 A. CRAIG, loc. cit.

35 R. SINGH, «Systems Theory in the Study of Industrial Relations: Time for a Reappraisal?», Industrial Relations Journal, vol. 7, no. 3, 1976, pp.59-71. 
- les intrants: buts, valeurs et pouvoir des parties influencés par l'environnement (systèmes écologique, économique, politique, légal et social);

- les mécanismes de conversion des intrants en extrants;

- les extrants comprenant les récompenses autant financières que sociales et psychologiques accordées aux travailleurs;

- un effet de rétroaction des extrants du système sur les sous-systèmes d'environnement et sur le système de relations industrielles lui-même.

Craig a ainsi illustré en schématisant son modèle, les liens et les séries d'influence entre tous les éléments qui le composent.

Dans le même ordre d'idée, Singh propose un modèle qui distingue les structures (qui correspondent aux acteurs de Dunlop) des processus de détermination des règles. Il inclut dans son modèle l'analyse du conflit autant que du consensus, répondant par là à une critique souventes fois formulée à l'égard du modèle de Dunlop quant à son incapacité à tenir compte du conflit comme variable importante du système.

Enfin, Sellier ${ }^{36}$, Gill \& Concannon ${ }^{37}$, Crispo ${ }^{38}$ et Wood et al. ${ }^{39}$, retiennent essentiellement la même variable dépendante que Dunlop, soient les règles. D'autres auteurs, à l'instar de Blain et Gennard retiennent plutôt les processus de création des règles: c'est le cas notamment de Hameed ${ }^{40}$, de Dabscheck $^{41}$, de Purcell \& Earl ${ }^{42}$ et de Parker \& Scott ${ }^{43}$.

Le système de Dunlop est organisé en fonction de règles et de consensus. Ce biais, introduit par l'application que Dunlop a voulu faire du modèle parsonien, (plutôt statique et qui considère le conflit et les structures

36 F. SELLIER, $L$ 'analyse systémique et les relations industrielles, texte miméographie, non daté.

37 C.G. GILL \& H.M.G. CONCANNON, «Developing an Explanatory Framework for Industrial Relations Policy within the Firm», Industrial Relations Journal, vol. 7, no 4, 1976-1977, pp. 13-21.

38 John H.G. CRISPO, Industrial Relations - Challenges and Responses, Toronto, University of Toronto Press, 1966.

39 S.J. WOOD, A. WAGNER, E.G.A. ARMSTRONG, J.F.B. GOODMAN \& J.E. DAVIS, "The Industrial Relations System Concept as a Basis for Theory in Industrial Relations», British Journal of Industrial Relations, vol. XIII, no. 3, 1975, pp. 291-308.

40 S.A. HAMEED, «A Critique of Industrial Relations Theory», Relations industrielles, vol. 37, no. 1, 1982, pp. 15-30; "A Theory of Responsive Bargaining", Labor Law Journal, vol. 24, no. 8, 1973, pp. 550-558; "Theory and Research in the Field of Industrial Relations», British Journal of Industrial Relations, vol. V, no. 2, 1967, pp. 222-237.

41 Braham DABSCHECK, «The Australian System of Industrial Relations: An Analytical Model», The Journal of Industrial Relations, vol. 22, no. 2, juin 1980, pp. 196-218.

42 J. PURCELL \& J. Michael EARL, «Control Systems and Industrial Relations», Industrial Relations Journal, vol. 8, no. 2, 1977, pp. 41-54.

43 S.R. PARKER \& M.H. SCOTT, «Developping Models of Work Place Industrial Relations», British Journal of Industrial Relations, vol. IX, no. 2, July 1971, pp. 214-224. 
déviantes comme des éléments dysfonctionnels), a été à la source de nombreuses critiques sur l'utilité de l'approche systémique en relations industrielles.

Relativement au second courant de pensée, Dimitri Weiss considère les relations industrielles comme «l'ensemble des structures, mécanismes, opérations destinées à réguler le consensus social dans le système industriel» ${ }^{44}$. Ce point de vue, basé sur la recherche du consensus, souffre selon nous, de la même lacune que celle reprochée à Dunlop. Weiss cependant retient comme objet d'étude de cette "discipline», «l'analyse et la compréhension du travail sous tous ses aspects» ${ }^{45}$. Cette définition, bien que reconnue par l'auteur lui-même comme étant large, a cependant l'avantage de proposer un objet d'étude soit «le travail».

À l'opposé, Caire définit les relations industrielles «... comme l'étude des relations conflictuelles qui s'établissent entre groupes organisés à propos du travail dans les sociétés touchées par l'industrialisation» ${ }^{46}$. Cette définition amène une restriction de l'objet d'étude, le situant au seul niveau du groupe. Elle laisse ainsi de côté le niveau d'analyse individuel associé aux différentes facettes de la gestion des ressources humaines.

Margerison ${ }^{47}$ est certainement l'auteur qui insiste le plus sur l'aspect conflictuel des relations industrielles. Il suggère en effet que la nature et le développement du conflit constitue le coeur des relations industrielles... (Dunlop privilégiait le consensus). Margerison ajoute cependant que l'étude des relations d'emploi constitue une variable très importante en relations industrielles. Il s'agit là d'une vision plus large qui rejoint les conceptions de Heneman et Somers.

Heneman définit plus spécifiquement ce qu'il considère comme l'objet d'étude des relations industrielles lorsqu'il avance que cette discipline «is concerned with employment relationships in an industrial economy... Its central characteristic or focus is employment, in all its aspects (micro and macro, individual and group): labor marketing, labor relations, personnel management and the like. $\rangle^{48}$

Même s'il reconnaît que les relations industrielles n'est pas la seule discipline qui s'intéresse à l'emploi (le droit, l'économique, la sociologie et la

44 D. WEISS, Relations industrielles, Paris, Sirey (2nd) 1980, p. 5.

45 Ibid., p. 3.

46 Guy CAIRE, Les relations industrielles, Paris, Dalloz, 1973, p. 13.

47 J. MARGERISON, "What do we Mean by Industrial Relations - A Behavioral Science Approach», British Journal of Industrial Relations, vol. VII, no. 2, July 1969, pp. 273-286.

48 H.G. HENEMAN, «Toward a General Conceptual System of Industrial Relations: How do we get there», in G.G. Somers (Ed.) op. cit., p. 4. 
psychologie s'y intéressent également), Heneman souligne que c'est cependant la seule qui en fait son centre d'intérêt ce qui est suffisant pour en faire une discipline autonome. Boivin et Sexton abondent d'ailleurs dans ce sens lorsqu'ils affirment que l'on peut considérer les relations industrielles non pas comme une science fondamentale mais «comme un champ de connaisance dérivée, appliquant des éléments empruntés à diverses disciplines à l'examen d'une série particulière de problèmes reliés au travail, et jugé assez important pour que son étude soit érigée en discipline autonome».49

Somers reconnaît également l'apport d'autres disciplines en relations industrielles. Il croit cependant que l'essence des relations industrielles «is in the worker and his interaction with other workers and management at the workplace» ${ }^{50}$. Somers considère ainsi toutes les variables (économiques, sociologiques, psychologiques...) qui peuvent affecter cette relation d'emploi. Il retient comme niveau privilégié d'intérêt le lieu de travail immédiat du travailleur.

Même si Heneman et Somers s'expriment en des termes quelque peu différents pour définir leur variable dépendante, il n'en reste pas moins que leurs conceptions se rejoignent, la principale différence résidant dans le niveau d'analyse retenu. Ainsi Somers situe la variable dépendante d'un système de relations industrielles au niveau "micro» alors qu'Heneman privilégie la macro-analyse. Au-delà de cette différence, l'objet d'étude reste le même soit les relations d'emploi et l'accent est placé sur le travailleur.

Pour bien illustrer la pertinence de la variable retenue par Heneman ainsi que l'approche qu'il utilise, il est intéressant de se référer au modèle de prévision de main-d'oeuvre développé par Nystrom et Milkovich ${ }^{51}$, qui constitue en fait une application de l'approche retenue par Heneman.

En retenant comme variable dépendante les besoins de main-d'oeuvre, comme variable indépendante la demande du produit et comme variables modératrices, la structure de l'organisation et la possibilité de substitution technologique, ces auteurs montrent schématiquement l'utilité de l'approche systémique dans l'identification de variables importantes empruntées à plusieurs disciplines pour comprendre des problèmes à caractère multidisciplinaire.

49 J. BOIVIN, J. SEXTON, Les relations industrielles, Québec, Université Laval, Département des relations industrielles, notes de cours, juillet 1981, p. 56.

50 G.G. SOMERS, «Bargaining Power and Industrial Relations Theory», in G.G. Somers (Ed.) op. cit., p. 44.

51 G.T. MILKOVICH, P. NYSTROM, «Manpower Planning and Interdisciplinary Methodologies», Manpower and Applied Psychology, vol. 2, no. 2, 1968, pp. 17 à 21 . 
Quant aux Webb ${ }^{52}$ ils assimilent les relations industrielles à la négociation collective. Laffer ${ }^{53}$ pour sa part, retient comme objet d'étude les relations de négociation entendues dans un sens plus large que celui des Webb.

Toutes ces définitions ne sont cependant pas exclusives. On se rend ainsi compte que la création des règles, entendues au sens strict, constitue un sous-produit particulier de la négociation collective qui ne constitue elle qu'un aspect particulier de la relation d'emploi. Force est quand même de constater qu'en dépit des améliorations apportées par différents auteurs dans l'identification des variables indépendantes, pertinentes aux relations industrielles, on ne retrouve pas encore de consensus sur la variable dépendante (objet - «out put») propre aux relations industrielles.

\section{Les relations}

Quelques auteurs seulement semblent accorder de l'importance aux relations qui doivent exister entre les différentes variables d'un système de relations industrielles. Ainsi Heneman retient des relations de type causal rejoignant Singh qui souligne win essence, all a system model is trying to do is to explain cause and effect» ${ }^{54}$. De même, Blain et Gennard semblent privilégier ce type de relations, même si leur modèle n'offre que peu de précision à ce sujet. Gill \& Concannon, Hameed, Parker et Scott, Nystrom et Milkovich se rangent également derrière cette approche.

Ce choix s'oppose à celui de plusieurs théoriciens des systèmes qui privilégient plutôt les relations de type circulaire. Seul Craig retient sans plus de précisions cependant, des relations à causalité multiple.

Il est assez étonnant de constater que cet important aspect d'un système de relations industrielles soit ignoré par la plupart des théoriciens en relations industrielles encore que cette lacune soit compréhensible dans le cas de quelques-uns dont Laffer qui ne voit pas l'utilité de se servir du concept de système en relations industrielles et Sellier qui ne retient pas non plus cet aspect.

\section{L'aspect opérationnel}

Relativement à cet aspect des relations industrielles, Heneman est le seul à démontrer l'existence des relations dynamiques existant entre les systèmes conceptuels et opérationnels. Singh, Nystrom \& Milkovich ainsi que Parker et Scott reprennent essentiellement les idées de Heneman sur ce point particulier.

52 S. WEBB, B. WEBB, Industrial Democracy, New York, Longmans, 1970.

53 K. LAFFER, «Is Industrial Relations an Academic Discipline», The Journal of Industrial Relations, vol. 16, no. 1 , mars 1974 , pp. 62-73.

54 SINGH, op. cit., p. 69. 
On doit quand même souligner l'effort fourni par Blain et Gennard pour donner une forme mathématique au modèle amélioré de Dunlop, rendant ainsi théoriquement possible, même si ces auteurs ne s'astreignent pas à cette gymnastique, la mesure des relations entre variables. Il apparaît important de souligner ici qu'un certain nombre de variables étudiées dans le contexte des relations industrielles auraient avantage à être précisées, bref à être «mesurées» ceci dans le but d'en arriver à une meilleure compréhension des phénomènes qu'elles soustendent. Ce n'est qu'en s'astreignant à une telle discipline qu'il sera possible d'en arriver à façonner le contenu d'un système de relations industrielles.

\section{L'aspect dynamique}

Selon la définition retenue précédemment, un système est dynamique s'il prévoit la possibilité de changement dans les variables retenues et surtout dans leurs relations. Bien sûr, Heneman, Singh, Somers, Gill \& Concannon, et de façon générale, tous les auteurs qui ont traité de cet aspect, affirment qu'un système de relations industrielles est essentiellement dynamique. Ils ne démontrent cependant pas comment ils respectent cette caractéristique.

Quant à Blain et Gennard, ils soulignent que le fait de comparer leur système de relations industrielles à deux moments différents le rend dynamique.

\section{Système ouvert, fermé}

Seuls Singh et Craig précisent qu'un système de relations industrielles ouvert est influencé par son environnement et l'influence en retour rejoignant ainsi Katz et Kahn. La plupart des auteurs traitent bien sûr, plus ou moins rapidement, de l'influence de différents contextes sur le système de relations industrielles laissant entendre par là qu'il s'agit d'un système ouvert.

Quant à la présence de différents niveaux d'analyse, elle a surtout été précisée par Heneman et Somers. On retrouve aussi certains auteurs tels Hameed et Parker \& Scott qui identifient deux niveaux de systèmes, micro et macro, mais sans développer davantage cet aspect.

On peut ainsi à l'instar d'Heneman, retenir trois niveaux distincts d'analyse: celui de l'individu, celui des groupes et celui de la société. Ainsi la variable dépendante «main-d'oeuvre» retenue par Heneman, pour caractériser les relations industrielles se situe au niveau le plus global soit celui de la société. On la retrouve également exprimée différemment aux 
niveaux des groupes ou de l'individu. Il est donc possible et même essentiel de retenir plusieurs variables dépendantes selon le niveau auquel on s'adresse. Ces différentes variables conceptuelles qui sont le centre d'intérêt de systèmes partiels de relations industrielles doivent être reliées entre elles ce qui mène à l'intégration des systèmes partiels avec le système global de relations industrielles.

On ne peut nier en effet que la satisfaction au travail, par exemple, aura un sens différent selon qu'elle sera associée à un individu, un groupe ou une société. De plus, les variables indépendantes seront différentes ou tout au moins joueront différemment selon le niveau considéré.

On n'insistera jamais assez sur l'importance de bien distinguer le niveau auquel s'adresse un système de relations industrielles. Cette faiblesse que l'on retrouve chez beaucoup d'auteurs à commencer par Dunlop qui soutenait que son modèle pouvait s'appliquer à tous les niveaux, est certainement une des causes de la confusion qui entoure encore l'objet d'étude des relations industrielles.

\section{La validité}

Cette dernière caractéristique ne semble pas susciter beaucoup d'intérêt chez les théoriciens des relations industrielles. Quand on y fait allusion, on relie la validité du système à sa capacité à rendre compte adéquatement de la réalité. On fait également référence au pouvoir de prédiction d'un modèle. Force est de constater que sur ce plan, la «théorie» des relations industrielles accuse des lacunes très prononcées quant à sa capacité de prédire ou tout au moins d'expliquer certains phénomènes qui caractérisent le monde du travail.

Un tableau analytique regroupant la position des auteurs qui se sont prononcés sur les différentes caractéristiques propres aux relations industrielles peut être obtenu en s'adressant aux auteurs de l'article. La synthèse de ce tableau permet de nous rendre compte de la faiblesse qui existe quant à la compréhension de ce concept ainsi que de son application aux relations industrielles. Malgré ce fait, la revue de littérature faite, nous permet de dégager certaines caractéristiques d'un système de relations industrielles.

$1^{\circ}$ Une première constatation que l'on peut faire est que les relations industrielles constituent un champ d'étude qui manque de cohérence particulièrement au niveau théorique. Cette faiblesse apparaît évidente du fait que les auteurs ne s'entendent pas quant à l'objet d'étude même des relations industrielles; 55

55 Milton DERBER, «Comment on Collective Bargaining and Industrial Relations by Thomas A. Kochan», Industrial Relations XXI, Winter, 1982, pp. 84-91. 
$2^{\circ}$ Comme le souligne Hyman $^{56}$, cette constatation est sans doute due au fait que le caractère propre à cette matière dérive de préoccupations pratiques concernant une série de problèmes avec lesquels se sont trouvés confrontés les «acteurs» de Dunlop, c'est-à-dire les salariés, employeurs et l'État. Il fallait donc trouver rapidement des solutions aux problèmes qui caractérisaient le monde du travail des années d'après-guerre;

$3^{\circ}$ On comprend alors pourquoi la notion de «système de relations industrielles» mise de l'avant par Dunlop, a reçu tant d'attention. La proposition de Dunlop était à l'effet de faire du concept de «système de relations industrielles» le noyau théorique d'une future discipline qui serait cohérente et autonome. Il n'est donc pas surprenant que la grande majorité des auteurs étudiés adaptent son approche fonctionnaliste ainsi que l'objet («out put») qu'il préconisait soit la création de règles gouvernants les acteurs du système. En d'autres mots, selon cette perception, les relations industrielles peuvent être considérées «comme une série d'institutions stables, à travers lesquelles les «inputs» des buts et intérêts divergents sont transformés très normalement en un «réseau de règles» qui sous-tendent la progression sans heurts de la production capitaliste; ${ }^{57}$

$4^{\circ}$ En opposition à ce premier courant de pensée, on décèle un second groupe d'auteurs qui délaissent l'approche fonctionnaliste et optent plutôt pour une approche qui vise à préciser un objet d'étude autour duquel se greffe une multitude d'éléments qui permettent d'expliquer les variations de cet objet. Chez ces auteurs, on détecte certains objets d'étude (variables dépendantes) qui sont avancés pour caractériser le «coeur» d'un système de relations industrielles. Ces éléments-clefs sont: la négociation collective, la main-d'oeuvre et le conflit;

$5^{\circ}$ Finalement, il est possible d'identifier un troisième groupe d'auteurs qui bien que ne retenant pas l'approche systémique, proposent comme "raison d'être» des relations industrielles, les relations qui prennent place entre employeurs et employés en matière de conditions $d u$ travail; 58

56 Richard HYMAN, «La théorie des relations industrielles: une analyse matérialiste», Sociologie du travail, vol. 21, no. 4, 1979, p. 419.

57 Ibid., pp. 421-422.

58 Roy J. ADAMS, Competing Paradigms in Industrial Relations, Hamilton, McMaster University, (Working Paper no. 190), April 1981, 27 pages. Dans cet ouvrage, l'auteur démontre que les relations industrielles ont été marquées par quatre (4) «Écoles» de pensée: 1) École du marché du travail; 2) École du management; 3) École «politique» et 4) l'École institutionnelle. 
$6^{\circ}$ Quant aux autres caractéristiques des systèmes de relations industrielles «proposés», force est de constater que les auteurs y accordent peu d'importance. Ceux qui y consacrent de l'attention considèrent qu'un système de relations industrielles doit être ouvert (existence de niveaux), essentiellement dynamique, permettant des relations de causalité simple et dont la validité s'établit par confrontation avec la réalité et la capacité de prédiction.

Cette revue de littérature nous incite à proposer quelques considérations relatives à l'élaboration d'un système de relations industrielles. Le schéma qui suit présente cette tentative qui tient compte des principales caractéristiques déjà énoncées.

Graphique 3

Modele d'un systeme de relations industrielles

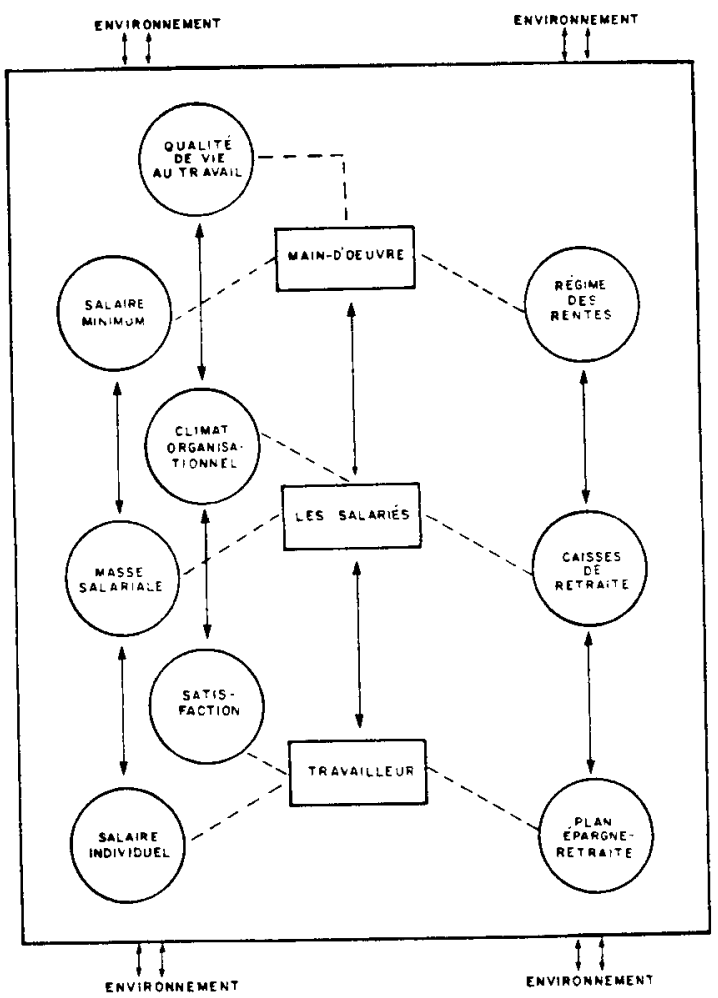


Pour les fins de la présentation, nous avons distingué trois niveaux d'analyse et conséquemment, nous avons retenu trois variables dépendantes ${ }^{59}$ qui se trouvent identifiées:

- au niveau individuel (ou micro): l'objet d'étude retenu est le travailleur dans son entité. Le terme travailleur désigne «un individu qui accomplit une activité physique ou intellectuelle quelconque moyennant rémunération ou autre forme de compensation".

- au niveau du groupe (ou organisationnel): la variable dépendante à ce niveau est constituée de l'ensemble des salariés (ou encore les ressources humaines) constituant l'ensemble des personnes qui travaillent pour une entreprise ou une organisation.

- au niveau de la société (ou macro): la main-d'oeuvre est la variable retenue. Elle est définie comme étant l'ensemble de tous les travailleurs. Il s'agit, en d'autres mots, de la force de travail d'une nation, d'un pays ou d'une province.

On pourrait sans doute retenir des niveaux supplémentaires d'analyse; tels ceux du secteur d'activité, ou encore du secteur géographique (région, etc...). Nous n'avons retenu que ces trois paliers parce qu'ils nous apparaissaient être les plus importants et qu'ils illustrent bien la différence et la complémentarité entre les différents systèmes de relations industrielles étudiant tour à tour le travailleur, les salariés d'une organisation et l'ensemble de la main-d'oeuvre que possède une nation. Chaque niveau constitue un système partiel avec sa propre variable dépendante (output) et son champ d'application particulier.

La reconnaissance des niveaux d'analyse suggère non seulement qu'un système de relations industrielles est ouvert (l'environnement), mais qu'il est également dynamique (les ressources - inputs ou entrées) puisqu'il existe en effet des relations dynamiques entre les systèmes partiels. L'ouverture est également représentée par les influences réciproques de l'environnement et du système de relations industrielles.

La seconde étape consiste à identifier quelques variables indépendantes qui doivent être en relation avec la variable dépendante retenue. La relation privilégiée ici, comme l'indique bien le modèle, est la relation de causalité simple.

Nous avons retenu, à titre d'exemple, trois variables indépendantes que l'on retrouve à chaque niveau. On remarque que chaque variable est libellée de façon différente selon le niveau considéré. Ainsi le salaire, au niveau in-

59 Le lecteur comprendra qu'il s'agit de la même variable dépendante identifiée différemment selon qu'elle est considérée à l'un ou l'autre des trois (3) niveaux d'analyse retenus. 
dividuel, correspond à la masse salariale au niveau organisationnel et au salaire minimum au niveau de la société.

Le lecteur comprendra que le lien qui unit chaque variable indépendante avec la variable dépendante correspondante (les activités du système) peut donner prise à la formulation d'une hypothèse qui demande à être testée. À titre d'exemple on pourrait affirmer que plus le travailleur a d'opportunité pour organiser son travail, plus il a de chance d'être satisfait (la satisfaction étant considérée comme une variable importante qui influence le travailleur - niveau individuel).

Ce dernier type de relation constitue en fait une hypothèse de recherche et peut se rattacher à une théorie de la satisfaction au travail. On illustre ainsi en même temps la démarche à suivre pour passer du niveau conceptuel au niveau opérationnel (la rétroaction ou le retour d'information) selon le schéma présenté dans la première partie de cet article (système, modèle, théorie, hypothèse, faits).

La précision des relations constitue une mesure de la validité du modèle conceptuel.

Le système étant essentiellement dynamique, il existe la possibilité d'inclure à tout moment dans le modèle toute autre variable indépendante ayant une relation avec la variable dépendante.

Le lecteur comprendra que l'objectif poursuivi ici est de proposer une démarche pour en arriver à «développer» la discipline des relations industrielles plutôt que de proposer un système comme tel de relations industrielles. Dans cette perspective, les auteurs sont bien conscients qu'il ne s'agit que d'une ébauche d'un système de relations industrielles. Malgré cette lacune, il apparaît fondamental d'en préciser les principales parties avant d'y ajouter d'autres caractéristiques telles que les rôles des différents acteurs qui interviennent dans un tel modèle ainsi que les règles qui affectent les comportements des différents intervenants qui y sont impliqués.

Dans la mesure où les personnes intéressées par les relations industrielles oeuvreront dans une perspective systémique, y insérant connaissances, expériences et découvertes, les phénomènes propres à ce champ d'études deviendront plus connus et, par voie de conséquence, plus faciles à prédire. 
Après tout, l'objectif visé, n'est-il pas de faire passer les relations industriel-

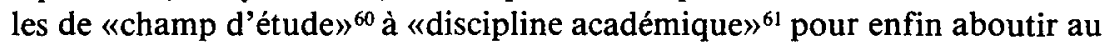
statut de «science» ${ }^{62}$.

\section{BIBLIOGRAPHIE}

ADAMS, R.J., Competing Paradigms in Industrial Relations, Hamilton, McMaster University, (Working Paper no. 190), April 1981, 27 pages.

ANDERSON, John C., «Bargaining Outcomes: an IR System Approach». Industrial Relations, vol. 18, no. 2, 1979, pp. 127-143.

BAIN, G.S. \& H.A. CLEGG, «A Strategy for Industrial Relations Research in Great Britain", British Journal of Industrial Relations, vol. XII, no. 1, 1974, pp. 91-113.

BARBASH, J., "The Elements of Industrial Relations», British Journal of Industrial Relations, vol. 11, no. 1, 1964, pp. 66-78.

BARKIN, Solomon, «European Industrial Relations. A Resource for the Reconstruction of the American System», Relations Industrielles, vol. 35, no. 3, 1980, pp. 439-445.

BEHREND, Hilde, "The Field of Industrial Relations", British Journal of Industrial Relations, vol. 1, no. 3, 1963, pp. 383-394.

BÉLANGER, Laurent, Gestion des ressources humaines, Chicoutimi, Gaétan Morin, 1978, 363 pages.

BERRIEN, K., General and Social Systems, Boston, Rutgers University Press, 1968, 231 pages.

BERTALANFFY, Von L., Théorie générale des systèmes, Paris, Dunod, 1973, 296 pages.

BLAIN, A.N.J. \& J. GENNARD, «Industrial Relations Theory: A Critical Review», British Journal of Industrial Relations, vol. VIII, no. 3, Novembre 1970, pp. 389-407.

60 Renvoie à une branche du savoir qui tend à délimiter les bornes de son étendue et à articuler sa démarche autour d'une méthodologie qui lui est propre.

61 Désigne une branche du savoir plus systématique, capable de questionner la réalité et de colliger des données d'une manière plus certaine justement parce qu'elle repose sur une méthodologie précise et déjà rodée.

62 Désigne un champ intellectuel pour lequel l'on dispose d'un ensemble de connaissances acquises au moyen d'une méthodologie propre et systématisée par rapport à un objet clairement identifié; l'organisation de ces connaissances et la compréhension des phénomènes qui lui sont propres, devraient permettre d'effectuer des prédictions pertinentes et bien appuyées au plan théorique. DELORME, op. cit., p. 2. Cette perspective est également mise en évidence par Roy J. ADAMS, op. cit., p. 4. 
BOIVIN, Jean \& Jacques GUILBAULT, Les relations patronales-syndicales au Québec, Chicoutimi, Gaétan Morin, 1982, 309 pages.

BOIVIN, J., J. SEXTON, Les relations industrielles, Québec, Département des relations industrielles, Université Laval, note de cours, juillet 1981, 487 pages.

BOUVIER, Émile, Les relations du travail au Québec, Montréal, Guérin, 1980, 403 pages.

BUCKLEY, Walter, Sociology and Modern Systems Theory, New-York, PrenticeHall, 1967, 227 pages.

CAIRE, Guy, Les relations industrielles, Paris, Dalloz, 1973, 112 pages.

CRAIG, Alton W., "A Model for the Analysis of Industrial Relations», H.C. Jain (ed), Canadian Labour and Industrial Relations Public and Private Sectors, Toronto, McGraw-Hill, 1974, pp. 2-12.

CRISPO, John H.G., Industrial Relations - Challenges and Responses, Toronto, University of Toronto Press, 1966, 156 pages.

DABSCHECK, Braham, "The Australian System of Industrial Relations: An Analytical Model», The Journal of Industrial Relations, vol. 22, no. 2, Juin 1980, pp. 196-218.

DAVISON, Robert B., «Industrial Relations Theory: A View from the Third World», Labor Law Journal, Vol. 24, no. 8, 1973, pp. 543-550.

DELORME, François, Élaboration d'un sous-système en relations industrielles, École de relations industrielles, Université de Montréal, (Texte miméographié), Décembre 1972, 87 pages.

_____ Étude empirique de la satisfaction au travail chez les contremaîtres québécois, Montréal, École de relations industrielles, Université de Montréal, (Thèse de maîtrise), 1974, 324 pages.

DERBER, M., «Comment on Collecting Bargaining and Industrial Relations by Thomas A. Kochan», Industrial Relations, XXI, Winter 1982, pp. 84-91.

DION, Gérard, Dictionnaire canadien des relations du travail, Québec, P.U.L., 1976, 662 pages.

- - - - «La recherche en relations industrielles dans les Universités du Québec», La recherche au Canada français, Montréal, Presses de l'Université de Montréal, 1968, pp. 71-86.

DOMINGUEZ, J.R., «An Analysis of the Industrial Relations System in a Collective Society", British Journal of Industrial Relations, Vol. IX, no. 1, March 1971, pp. 21-32.

DUNLOP, John T., Industrial Relations Systems, New-York, Henry Holt \& Co., 1958, 339 pages.

FATCHETT, Derek \& W.M. WHITTINGHAM, «Trends and Developments in Industrial Relations Theory», Industrial Relations Journal, Vol. 7, no. 1, 1976, pp. 50-61.

FLANDERS, Allan, «Industrial Relations: What is Wrong with the System?», Management and Unions, The Theory and Reform of Industrial Relations, Faber \& Faber, London, 1970, pp. 83-129. 
FORRESTER, Jay W., «Industrial Dynamics-After the First Decade», Management Science, Vol. 14, no. 7, March 1968, pp. 398-415.

GEARE, A.I., "The Field of Study of Industrial Relations», The Journal of Industrial Relations, Vol. 19, no. 3, September 1977, pp. 274-285.

GILL, J., «One Approach to the Teaching of Industrial Relations», British Journal of Industrial Relations, Vol. VII, no. 2, 1969, pp. 265-271.

GILL, C.G. \& H.M.G. CONCANNON, «Developing an Explanatory Framework for Industrial Relations Policy within the Firm», Industrial Relations Journal, Vol. 7, no. 4, 1976-1977, pp. 13-21.

DE GIVRY, J., Les relations industrielles: un point de vue international, (Conférence prononcée à l'occasion du 10e anniversaire de la Corporation des conseillers en relations industrielles du Québec), Montréal, 25 janvier 1974, 12 pages.

GOODMAN, J.F.B. et al, «Rules in Industrial Relations Theory: a Discussion», Industrial Relations Journal, Vol. 6, no. 1, 1975, pp. 14-30.

HAMEED, Syed M.A., «A Critique of Industrial Relations Theory», Relations industrielles, Vol. 37, no. 1, 1982, pp. 15-30.

no. 8, 1973, pp. 550-558.

, "Theory and Research in the Field of Industrial Relations», British Journal of Industrial Relations, Vol. V, no. 2, 1967, pp. 222-237.

HENEMAN, Herbert G., "Conceptual System of Industrial Relations», Manpower and Applied Psychology, Vol. 1 et 2, 1967, pp. 95-101.

, «Contributions of Industrial Relations Research», Manpower and Applied Psychology, Vol. 2, no. 2, 1968, pp. 5-16.

_-___- «Toward a General Conceptual System of Industrial Relations: How do we get there?», G.G. Somers (ed), Essays in Industrial Relations Theory, Iowa, Iowa State University Press, 1969, pp. 3-24.

-___- "Toward a System of Systems», Manpower and Applied Psychology, Vol. 3, nos 1 et 2, 1969, pp. 37-41.

HENEMAN, H.G. \& D. YODER, Labor Economics, Ohio, South Western Publishing Co., 1965, 824 pages.

HILL, S. \& K. THURLEY, «Sociology and Industrial Relations», British Journal of Industrial Relations, Vol. XII, no. 2, July 1974, pp. 147-170.

HYMAN, Richard, «La théorie des relations industrielles: une analyse matérialiste», Sociologie du travail, Vol. 21, no. 4, 1979, pp. 418-438.

HYMAN, Richard \& Ian BROUGH, Social Values and Industrial Relations. A Study of Fairness and Inequality, Oxford, Basil Blackwell, 1975, 277 pages.

KASSALOW, E.M., «Conflict and Cooperation in Europe's Industrial Relations», Industrial Relations, Vol. 13, no. 2, 1974, pp. 156-164.

KATZ, D. \& R.L. KAHN, The Social Psychology of Organisation, New-York, Wiley \& Sons, 1966, 498 pages.

KERR, Clark, «Industrial Relations Research: a Personal Retrospective», Industrial Relations, Vol. 17, no. 2, 1978, pp. 131-142. 
KERR, C. \& J.T. DUNLOP, P. HARBISON \& C.A. MYERS, Industrialism and Industrial Man, Cambridge, Harvard University Press, 1960, 331 pages.

KOCHAN, T.D., Collective Bargaining and Industrial Relations, Homewood, Ill., Irwin, 1980.

LAFFER, K., «Is Industrial Relations an Academic Discipline?», The Journal of Industrial Relations, Vol. 16, no. 1, 1974, pp. 62-73.

____ - «Les relations professionnelles - Enseignement et étendue du sujet: une expérience australienne», Bulletin de l'Institut international d'études sociales, Vol. 5, Novembre 1968, pp. 10-30.

, "Where Are we Going in Industrial Relations», The Journal of Industrial Relations, Vol. 18, no. 3, Septembre 1976, pp. 283-293.

MARGERISON, J., «What do we Mean by Industrial Relations - A Behavioral Science Approach», British Journal of Industrial Relations, Vol. VII, no. 2, July 1969, pp. 273-286.

MILKOVICH, G.T., «Toward a System of Systems», Manpower ad Applied Psychology, Vol. 3, nos 1 et 2, Winter 1969, pp. 37-41.

MILKOVICH, G.T. \& P.C. NYSTROM, «Manpower Planning and Interdisciplinary Methodologies», Manpower and Applied Psychology, Vol. 2, no. 2, 1968, pp. 17-21.

MOLHUYSEN, P.C., "The Academic Respectability of Industrial Relations and Labour Economics», The Journal of Industrial Relations, Vol. 17, no. 1, 1975, pp. 97-99.

MONGEAU, Pierre, «La pensée systémique. Historique et concepts», $L$ 'orientation professionnelle, vol. 16, no. 6, avril 1981, pp. 33-50.

- - - «La pensée systémique. Les concepts. Conclusion et perspectives», $L$ 'orientation professionnelle, vol. 17, no. 1, juin 1981, pp. 9-52.

MOTH, L.G., «Industrial Relations in the Plant - A Study of Evolution within the System», The Journal of Industrial Relations, Vol. 14, no. 3, 1972, pp. 282-293.

NEAL, C.B.E. \& F. LEONARD, «Future Trends in Industrial Relations», Occupational Psychology, Vol. 45, nos 3-4, 1971, pp. 167-172.

OWEN, W.V. \& Howard V. FINSTON, Industrial Relations, New-York, AppletonCentury-Crofts, 1964, 339 pages.

OXNAM, D.W., "Industrial Relations and Anzaas», The Journal of Industrial Relations, Vol. 16, no. 1, March 1974, pp. 74-77.

PARKER, S.R. \& M.H. SCOTT, «Developping Models of Work Place Industrial Relations», British Journal of Industrial Relations, Vol. IX, no. 2, July 1971, pp. 214-224.

PARSONS, Talcott, The Social System, New-York, The Yale Press, 1951, 540 pages.

PEACH, David A. \& David KUECHLE, The Practice of Industrial Relations, Toronto, McGraw-Hill, 1975, 358 pages.

PURCELL, John \& J. Michael EARL, «Control Systems and Industrial Relations», Industrial Relations Journal, Vol. 8, no. 2, 1977, pp. 41-54. 
REYNAUD, J.D., «Les relations professionnelles et la négociation du changement», Bulletin de l'Institut international d'Études sociales, no. 9, 1972, pp. 3-18. ROBERTS, B.C., «Industrial Relations and the European Economic Community», Labor Law Journal, Vol. 24, no. 8, pp. 484-491.

SCHIENSTOCK, G., "Toward a Theory of Industrial Relations», British Journal of Industrial Relations, Vol. XIX, no. 2, July 1981, pp. 170-189.

SELLIER, François, L'analyse systémique et les relations industrielles, Texte miméographié, (non daté), 15 pages.

SHIMMIN, S. \& R. SINGH, «Industrial Relations and Organizational Behaviour: A Critical Appraisal», Industrial Relations Journal, Vol. 4, no. 3, Automn 1973, pp. 37-43.

SINGH, R., «Systems Theory in the Study of Industrial Relations: Time for a Reappraisal?», Industrial Relations Journal, Vol. 7, no. 3, 1976, pp. 59-71.

- _ _ - «Theory and Practice in Industrial Relations», Industrial Relations Journal, Vol. 9, no. 3, 1978, pp. 57-64.

SOMERS, G.G., «Bargaining Power and Industrial Relations Theory», G.G. Somers (ed), Essays in Industrial Relations Theory, Iowa, Iowa State University Press, 1969, pp. 39-55.

STRAUSS, George, «The Study of Conflict: Hope for a New Synthesis Between Industrial Relations and Organizational Behavior?», Reprint no. 417, University of California, 1977, pp. 329-337.

THOMPSON, M., "Applying a Theory of the Future of Industrial Relations to North America», Labor Law Journal, Vol. 24, no. 8, 1973, pp. 564-572.

WEBB, Sydney \& B. WEBB, Industrial Democracy, New-York, Longmans, 1970, 899 pages.

WEISS, Dimitri, Relations industrielles, Paris, Sirey (2e ed), 1980, 257 pages.

WOOD, Stephen, «Ideology in Industrial Relations Theory», Industrial Relations Journal, Vol. 9, no. 4, 1978-1979, pp. 42-56.

WOOD, S.J. \& A. WAGNER \& E.G.A. ARMSTRONG \& J.F.B. GOODMAN \& J.E. DAVIS, «The Industrial Relations System Concept as a Basis for Theory in Industrial Relations», British Journal of Industrial Relations, Vol. XIII, 1975, pp. 291-308.

YOUNG, Stanley, «Industrial Relations: A Paradigmatic Analysis», Relations Industrielles, Vol. 37, no. 1, 1982, pp. 32-50. 


\section{The Systems Approach in Industrial Relations}

This article considers the concept of an industrial relations system. First, the systems approach is described and its implications for the field of industrial relations are discussed. The treatment of each element of the systems approach, by different authors, is examined. Second, an attempt is made to apply these elements to industrial relations and to present a more complete view of this field of study.

The systems concept, which originated in the natural sciences, rapidly became a key concept in many disciplines. In some respects, the systems approach's appeal is due to its inherent notions of order and logic. The elements of the concept are: definition of the system; description of its parts; relations among the parts; the operational dimension of the system; its static-dynamic dimension; its closed-open dimension; validity. A system may be defined as a complex set of interrelated variables. Functionally speaking, these variables are commonly identified as dependent, independent and intervening variables. The dependent variable is the heart of the system. It is what we seek to explain through the independent variables. Intervening variables are those which affect relationships between dependent and independent variables.

The operational dimension refers to the distinction between operational and conceptual systems, as well as to the system's validity, defined as its capability to reflect reality adequately. The openness of a system may be defined as the continuous exchange between the system and its environment. This flow of exchanges is essential to the system's viability. This dimension also refers to the existence of levels of systems. Finally, the system's dynamism refers to its capability to adapt to potential changes. Table I summarizes the ideas developed by several authors regarding these characteristics of the systems approach.

Analysis of the systems concept as it is currently used by authors in the Industrial Relations (I.R.) field reveals considerable neglect of the various characterictics of the systems approach. (An analytical list of authors is available on request.) Despite this neglect and the lack of a common definition of the dependent variable for the industrial relations system, we can nevertheless arrive at a tentative characterization of an I.R. system. It appears to be concerned with the process of rules creation and particularly with collective bargaining, although a few authors consider «manpower» as a more adequate dependent variable. With respect to the other characteristics of the systems approach, an I.R. system appears to be dynamic (changes may occur in the relationships between variables and variables themselves may change), open (there are levels of systems) and allowing causal relationships. Finally, its validity rests upon the capability to reflect the real world and make predictions.

The authors offer a model of an I.R. system that takes into account the main dimensions of the systems approach. Their proposal is illustrated in Diagram I: manpower, the dependent variable, is defined differently for each level considered, 
namely the micro level (worker), the group level (salaried employees, all the workers of an organization) and the macro or societal level (manpower). The diagram also reflects the characterization of an I.R. system as open, dynamic and allowing for causal relationships. The precision of measure of the relationships is a measure of the conceptual model's validity.

The authors offer only a rough sketch of an. I.R. system that needs to be improved by both practitioners and theoreticians. Hopefully, this will allow Industrial Relations to become a discipline in its own right, rather than a field of investigations for researchers in various other disciplines.

\section{LA RÉDUCTION DE LA DURÉ DU TRAVAIL}

Introduction: René BOULARD, Jean-Paul DESCHÊCHES, Alain LAROCQUE, Claude RONDEAU - La réduction du temps de travail: un droit ou un privilège? Alain LAROCQUE - La signification du travail en 1980: émergence de valeurs nouvelles? Jean-Paul MONTMIMY - Commentaires: Ghislain DUFOUR, Norbert RODRIGUE -Le temps libéré: à quels coûts? Kimon VALASKAKIS - Commentaires: Lise POULINSIMON, Bernard FORTIN - Table ronde: Sommes-nous tous égaux face à la diminution du temps passé au travail? Gilles FERLAND, Jean-Marie HAMELIN, Pierre-Paul PROULX - Le temps passé au travail: un élément encore négociable? Gilles MASSE -Commentaire: Jean-Paul DESCHÊNES - La législation: consolidation ou innovation? Gilles BEAUSOLEIL, Hervé GAUTHIER - Commentaires: Luc M. LOCKWELL et Pierre LORTIE - La technologie: un substitut à la durée du travail? Monique FRAP. PIER-DESROCHERS - Table ronde: La réduction du temps passé au travail: un moyen de lutte contre le chômage? Fernand D'AOUST, André DEOM, Pierre HARVEY - La diminution du temps de travail: un phénomène inéluctable? Michel CROZIER.

ISBN 2-7636-6973-X

1 volume, 273 pages - Prix: $\$ 17.00$

Les Presses de l'Université Laval

Cité universitaire

C.P. 2447, Québec, P.Q., Canada, G1K 7R4 